\title{
La información científica en Internet vista por estudiantes de Educación Secundaria Obligatoria: Un estudio exploratorio de sus competencias digitales
}

\author{
Daniel Valverde-Crespo \\ I.E.S. Eduardo Linares Lumeras. Molina de Segura, Murcia. España. Departamento de Didáctica de las \\ Ciencias Experimentales, Facultad de Educación, Universidad de Murcia. Murcia. España. \\ daniel.valverde@um.es \\ ORCID: http:// orcid.org/0000-0003-4322-367X
}

Antonio de Pro-Bueno

Departamento de Didáctica de las Ciencias Experimentales, Facultad de Educación, Universidad de Murcia. Murcia.nono@um.es ORCID: http:// orcid.org/0000-0003-3301-8787

Joaquín González-Sánchez

Departamento de Quimica-Física, Facultad de Quimica, Universidad de Murcia.Murcia.josquin@um.es ORCID: bttp:// orcid.org/0000-0001-6848-074X

[Recibido: 21 Febrero 2019. Revisado: 13 Mayo 2019. Aceptado: 20 Septiembre 2019]

Resumen: En la actualidad Internet se ha convertido en la primera fuente de información científica. Esto justifica la necesidad de que todo ciudadano disponga de competencias que permitan evaluar los contenidos y su fiabilidad. En este trabajo se pretende explorar y describir las competencias digitales, adquiridas dentro o fuera del aula, que manifiestan un grupo de estudiantes de $4^{\circ}$ de ESO al valorar un texto de Internet sobre un tema científico. Los resultados indican que los participantes tienen un buen desempeño para identificar ideas y posicionarse respecto a la información. Sin embargo, tienen dificultades para hacer inferencias lejanas, localizar errores, interpretar información y calificar su fiabilidad. Los resultados respaldan la necesidad de desarrollar competencias en las aulas para formar ciudadanos capaces de ser críticos al evaluar la enorme cantidad de información a la que están expuestos.

Palabras clave: Competencia digital; Habilidades de información; Reacciones químicas; Aprendizaje de las ciencias; Educación Secundaria Obligatoria.

Internet scientific information seen by students of Compulsory Secondary Education: An exploratory study of their digital competences

\begin{abstract}
Nowadays, the Internet has become the first source of scientific information. This fact justifies the need for every citizen to have competences that allow the evaluation of contents and their reliability. This paper aims to explore and describe the digital competences, acquired inside or outside the classroom, which are manifested by a group of 4th year ESO students when they assess an Internet text on a scientific topic. The results indicate that the participants show good performance to identify ideas and position themselves regarding the information. However, they have difficulty making distant inferences, locating errors, interpreting information and rating their reliability. The results support the need to develop competences in classrooms to train citizens capable of being critical and objective when evaluating the enormous amount of information that they are exposed to.
\end{abstract}

Keywords: Digital Competence; Information skills; Chemical reactions; Science learning; Secondary Education.

Para citar este artículo: Valverde-Crespo D., Pro-Bueno A., González-Sánchez J. (2020) La información científica de Internet vista por estudiantes de Educación Secundaria Obligatoria: Un estudio exploratorio de sus competencias digitales. Revista Eureka sobre Enseñanza y Divulgación de las Ciencias 17(1), 1101. doi: 10.25267/Rev_Eureka_ensen_divulg_cienc.2020v17.11.1101 


\section{Introducción y objetivo de la investigación}

El uso de Internet como fuente de información se encuentra plenamente integrado en múltiples ámbitos de la vida cotidiana (ocio, trabajo, enseñanza...). Concretamente en España se ha convertido en la primera fuente de consulta sobre temas científicos (FECYT 2015) y esta realidad no es ajena nuestro alumnado, ya que los jóvenes entre 15 y 24 años son el grupo de edad que más utiliza esta herramienta, siendo las redes sociales, YouTube y Wikipedia los medios a los que más acuden (Revuelta y Corchero 2017).

Ahora bien, el uso habitual de la red para informarnos no está exento de riesgos. Como señalaba Monereo (2005), además de un conjunto de características positivas (accesibilidad, actualidad, interconectividad...), Internet posee otras que no lo son: la sobreabundancia informativa, los problemas de garantía y fiabilidad, la búsqueda desorientada, la información basura, los fines comerciales... Todas ellas deben ser tenidas en cuenta para su utilización.

Algunos datos resultan preocupantes en el ámbito de las Ciencias. Valga como ejemplo que uno de cada tres «bulos» presentes en Internet está relacionado con temas de salud (oncología, nutrición...) (EFE 2018). Probablemente por ello, una red social del tamaño de Facebook ha puesto en marcha una campaña contra la difusión de bulos y noticias falsas que diariamente se publican, proporcionando a sus usuarios directrices para detectar estas tergiversaciones y desinformaciones (Jiménez 2017). Este riesgo, en particular, hace necesario que el usuario disponga de pautas y habilidades para analizar o valorar de forma crítica la fiabilidad de la información de Internet.

Estas y otras habilidades forman parte de la competencia digital, una de las incluidas en el currículo oficial para la formación obligatoria de cualquier ciudadano. Ésta se define como: «Aquella que implica el uso creativo, crítico y seguro de las TIC para alcanzar los objetivos relacionados con el trabajo, la empleabilidad, el aprendizaje, el uso del tiempo libre, la inclusión y la participación en la sociedad» (BOE 2015, p.6995). Además, desde una perspectiva institucional, se debe contribuir a su desarrollo desde todas las materias curriculares, también desde las que tienen un carácter científico.

Ahora bien, con independencia de la obligatoriedad curricular, el interés en la competencia digital se debe a que es muy utilizada por los estudiantes en sus tareas académicas o en otras actividades cotidianas. Sin embargo, como han señalado algunas investigaciones (Grimalt, Pintó y Ametller 2013, Gómez, Cañas, Gutiérrez y Martín 2014, Area, Cepeda y Romero 2018), las TIC no se utilizan mucho en las clases de Ciencias y, cuando se hace, generalmente se realiza con fines expositivos y manejadas por el profesorado. Esto conlleva que su adquisición por los estudiantes suceda fundamentalmente fuera del contexto escolar, con lo que aprenden procedimientos y desarrollan capacidades -paradójicamente muchas veces desconocidas e ignoradas en la enseñanza- que podrían y deberían utilizarse en la alfabetización científica (Pereira, Fullol y Moura 2019). Por todo ello, es necesario conocer el grado de desarrollo de la competencia digital de nuestro alumnado, independientemente de su origen, como paso previo a tratar de actuar sobre la misma (Pedrinaci 2012a).

En un trabajo anterior (Valverde-Crespo, Pro-Bueno y González-Sánchez 2018), revisamos las investigaciones y propuestas en relación a esta competencia en el ámbito de la didáctica de las ciencias experimentales (DCE). Al respecto, destacábamos la importancia de estas contribuciones por la creciente cantidad de información científica existente en la red. Esto reforzaría la idea que los estudiantes deben disponer de los conocimientos necesarios para comprenderla o discutirla, pero también tienen que adquirir las capacidades digitales para identificar, filtrar y evaluar la información. 
Por ello, el objetivo que persigue este estudio es el siguiente:

- Describir qué competencias digitales ponen de manifiesto unos estudiantes de educación secundaria obligatoria (ESO) al utilizar y valorar la información de un texto, semejante a los que pueden encontrar en Internet, en el que subyacen unos contenidos científicos de química.

\section{Revisión de aportaciones}

A menudo, el profesorado y otros agentes educativos perciben la competencia digital de forma limitada. Por ello, nos parece adecuado definir qué entendemos por competencia digital. Esta investigación se apoya en el DIGCOMP: A Framework for Developing and Understanding Digital Competence in Europe (Ferrari 2013), que es el marco de referencia europeo para la formulación de políticas e iniciativas educativas para el desarrollo de dicha competencia. Al respecto, se distinguen cinco áreas: Información, Comunicación, Creación de contenido, Seguridad y Resolución de problemas.

Como se ha indicado, la problemática de este estudio se ubica en el área de información que implica: «Identificar, localizar, recuperar, almacenar, organizar y analizar la información digital, evaluando su relevancia y su propósito» (Ferrari 2013, p.4).

Esta área se divide en subcompetencias y nuestro estudio lo ubicamos en «Evaluación de información» que se define como: «Recuperar, procesar, comprender y evaluar críticamente la información» (Ferrari 2013, p.5). Por lo tanto, esta sub-competencia implica varias capacidades, que van desde la valoración de su credibilidad, propósito y relevancia hasta la comprensión y procesamiento de su contenido, lo que conlleva que el alumnado utilice sus conocimientos sobre la temática.

Por otro lado, hemos revisado las aportaciones sobre las competencias digitales de los adolescentes. Las investigaciones han mostrado que este colectivo tiene un nivel alto en las capacidades instrumentales de manejo de las TIC y en una serie de acciones o tareas a las que están habituados (Colás, Conde y Reyes 2017). Sin embargo, sus capacidades se reducen si aumenta la complejidad cognitiva de las competencias, mostrando niveles bajos en la seguridad online, los problemas sociales asociados a las TIC y al evaluar información (Li y Ranieri 2010, Calvani, Fini, Ranieri y Picci 2012, Somyurek y Coskun 2013).

La introducción de las TIC en las aulas en los últimos años tampoco ha supuesto una mejora en la competencia digital de los estudiantes, porque no ha sido acompañada de cambios necesarios en la metodología docente (Hatlevik y Christophersen 2013, Fernández 2016). Además, en relación a la valoración de la información sobre temáticas científicas por parte de estudiantes de secundaria (no solamente en formatos digitales), se ha encontrado que:

- No dudan de la información de forma espontánea (Calvani et al. 2012, Mason, Junyent y Tornatora 2014) ya que, durante su escolaridad, han recibido la información científica de fuentes que son consideradas una autoridad (profesores de ciencias, libros de texto...), avaladas socialmente (Valverde-Crespo y González-Sánchez 2016), por lo que no desarrollan una mirada crítica hacia su contenido.

- Muestran dificultades para explicitar sus ideas científicas, para localizar errores conceptuales o para detectar información contradictoria en textos (físicos o digitales) o en formatos audiovisuales (Otero y Campanario 1990, Keck, Kammerer y Starauschek 2015, Valverde-Crespo, González-Sánchez y Pro-Bueno 2017, Bravo, Bouciguez y Branmüller 2019). Además, se ha comprobado que un mayor conocimiento científico 
y una mejor capacidad lectora mejoran la evaluación de la información de Internet (Forzani 2018, Sullivan y Puntambekar 2019).

- No suelen valorar los resultados de las búsquedas que realizan en Internet y, en caso de hacerlo, usan elementos de poca profundidad como el título de la página web o el pequeño resumen que ofrece el buscador (Julien y Barker 2009, Walraven, BrandGruwel y Boshuizen 2009).

También es preciso destacar la complejidad que lleva la adquisición de esta competencia. Como señalan Colwell, Hunt y Reinking (2013), las estrategias y competencias previas en el manejo de información (búsqueda, comprensión, evaluación...) que tienen los estudiantes son de bajo nivel y suponen muchas veces un obstáculo -más que una ayuda- para el desarrollo escolar de un mayor nivel competencial, debido a que hay rutinas no deseables que están muy arraigadas, por ejemplo, copiar la información encontrada sin comprenderla, no cuestionarse datos contradictorios, leer sólo el primer enlace de una búsqueda, etc.

\section{Diseño del estudio}

Nuestro trabajo tiene un enfoque exploratorio-descriptivo. Describimos los participantes y el contexto, y el instrumento de recogida de información utilizado.

\section{Participantes y contexto}

El estudio fue realizado en tres centros públicos de secundaria de la ciudad de Murcia. Participaron 86 estudiantes de $4^{\circ}$ de ESO, con edades entre 14 y 16 años, que cursaban la materia de Física y Química. No hubo ningún criterio de selección previo. Los centros disponían de aulas de informática o de ordenadores portátiles con los que se llevó a cabo la experiencia.

Por otro lado, dado que queríamos identificar competencias digitales adquiridas por unos estudiantes ante una información de carácter científico, se eligió como temática las reacciones químicas por varias razones. Es un contenido relevante en la enseñanza obligatoria (Caamaño 1998) y un referente del currículo de la ESO (CARM, 2015), una idea central que favorece una interrelación entre múltiples contenidos escolares. De hecho, según los programas oficiales, al final de dicha etapa, el alumnado debería disponer de un modelo interpretativo, aunque construido e influenciado por múltiples dificultades (Solsona e Izquierdo 1999); entre ellas, las representaciones sociales de los estudiantes (Lacolla, Meneses y Valeiras 2013), condicionadas por su contexto cotidiano y por las múltiples informaciones que reciben de forma activa o pasiva por diferentes medios (Internet, televisión, publicidad...), a menudo de carácter quimiofóbico.

El estudio se realizó durante el primer trimestre del curso, antes de que los docentes impartieran el tema de las reacciones químicas; de esta manera tratamos de evitar que hubiera factores que influyeran en las respuestas o las condicionaran (contenido impartido, prioridades del profesor, recursos utilizados...). Todos habían cursado en $3^{\circ}$ de ESO los contenidos propuestos por la legislación oficial sobre reacciones químicas (CARM 2015).

\section{Descripción del recurso y del instrumento de recogida de información}

Individualmente cada estudiante accedió y leyó un texto (con el formato de Internet) y debió responder un cuestionario. El tiempo para ello fue el de una hora lectiva de clase (55 minutos). Se utilizó un texto elaborado por los autores, titulado «Reacciones químicas de combustión», que se ubicó en una plataforma de blogs (Blogger): infor-quimica.blogspot.com.es. Dicho texto se incluye en el Anexo I, aunque sin la interfaz del blog empleado. 
Se pretendía crear una situación similar a la que habitualmente encuentran al usar Internet, en la que pusieran en juego sus competencias. Es decir, como señala Monereo (2005), un texto así debería tener información errónea, fines comerciales, dudosa fiabilidad, etc., y el nuestro fue escrito con estos requisitos. Se introdujeron tres errores diferentes: gráfico, textual y numérico. Consideramos que deberían ser sencillos y llamativos para el alumnado de $4^{\circ}$ de ESO. Éstos eran:

- Error 1: Indicación incorrecta de los reactivos y productos en una ecuación química.

$$
\begin{gathered}
\mathrm{C}_{4} \mathrm{H}_{10}+\frac{13}{2} \mathrm{O}_{2} \rightarrow 4 \mathrm{CO}_{2}+5 \mathrm{H}_{2} \mathrm{O} \\
\text { PRODUCTOS REACTIVOS }
\end{gathered}
$$

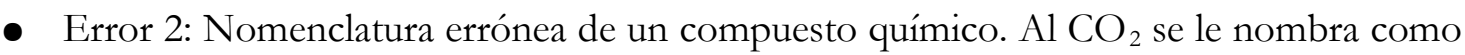
trióxido de carbono. Este compuesto es conocido y estudiado desde cursos tempranos, por lo que debería ser reconocido sin dificultad.

- Error 3: Dato erróneo de la emisión de $\mathrm{CO}_{2}$ per cápita en España durante el año 2011. Se dice que es $0,0000058 \mathrm{~kg}$ por persona, magnitud muy pequeña que debería ser valorada sin dificultad en $4^{\circ}$ de ESO. El dato real está en torno a 5,8 Tm (Banco Mundial 2016, OECD 2016).

Se informó a los participantes que existían errores ya que alertar de su presencia podía estimular y mejorar su localización (Keck et al. 2015).

En el último párrafo, se introdujo un mensaje publicitario sobre un producto ficticio que eliminaba el dióxido de carbono generado por los vehículos con motor de gasolina; se apoyaba en la afirmación de una empleada de la empresa que lo fabricaba. Con ello queríamos indagar en la capacidad para interpretar el propósito de una información ya que los bulos, falsedades o la publicidad son una oportunidad para ello (Olazabal y Echeverría 2013).

Por otro lado, el cuestionario empleado constaba de 9 cuestiones. Cada una pretendía explorar el grado de adquisición de unas subcompetencias digitales de tipo informativo. Adaptamos algunas ya utilizadas en otros trabajos (Pedrinaci 2012b, Pro-Bueno 2014, Valverde-Crespo, González-Sánchez y Pro-Bueno 2017, Cantó-Doménech, Pro-Bueno y Solbes 2017).

La Tabla 1 recoge las preguntas 1-6, sus subcompetencias y las características de las respuestas deseables.

Tabla 1. Cuestiones, competencias y respuestas deseables del cuestionario

\begin{tabular}{|l|l|l|}
\hline Cuestión & Subcompetencia & Respuesta deseable \\
\hline $\begin{array}{l}1 \text { - Según lo que has leído en la información, explica } \\
\text { brevemente qué es una reacción química de combustión. } \\
2 \text { - ¿Qué dos sustancias debe haber siempre para que } \\
\text { pueda ocurrir una reacción de combustión? }\end{array}$ & $\begin{array}{l}\text { Identificación de } \\
\text { ideas en el texto }\end{array}$ & $\begin{array}{l}\text { La respuesta deseable se encuentra } \\
\text { en texto de forma literal o } \\
\text { explícita. }\end{array}$ \\
\hline $\begin{array}{l}3 \text { - Además del ejemplo de la combustión del butano en un } \\
\text { calentador de agua y de la gasolina en un motor, indica } \\
\text { otros dos ejemplos donde podemos encontrar reacciones } \\
\text { químicas de combustión en la actividad humana. }\end{array}$ & $\begin{array}{l}\text { Inferencia lejana al } \\
\text { texto }\end{array}$ & $\begin{array}{l}\text { La respuesta deseable se relaciona } \\
\text { con la temática del texto, pero no } \\
\text { con el contenido del texto. }\end{array}$ \\
\hline $\begin{array}{l}4 \text { - Si las reacciones de combustión son tan perjudiciales } \\
\text { realizando actividades donde tienen lugar estas reacciones? }\end{array}$ & $\begin{array}{l}\text { Posicionamiento } \\
\text { argumentado }\end{array}$ & $\begin{array}{l}\text { La respuesta deseable debe apoyar o } \\
\text { discutir la información del texto y } \\
\text { hacerlo con argumentos. }\end{array}$ \\
\hline $\begin{array}{l}5 \text { - En el texto hay tres errores, ¿puedes decir cuáles son? } \\
\text { Por qué son errores? }\end{array}$ & $\begin{array}{l}\text { Localización de } \\
\text { errores }\end{array}$ & $\begin{array}{l}\text { La respuesta deseable identifica y } \\
\text { justifica los errores en el texto. }\end{array}$ \\
\hline $\begin{array}{l}6 \text { - ¿Qué crees que pretende la autora al escribir y publicar } \\
\text { la información del último párrafo del texto? ¿Por qué } \\
\text { piensas eso? }\end{array}$ & $\begin{array}{l}\text { Interpretación de } \\
\text { intencionalidad de la } \\
\text { información }\end{array}$ & $\begin{array}{l}\text { La respuesta deseable debe apoyarse } \\
\text { en el texto para identificar su } \\
\text { intencionalidad. }\end{array}$ \\
\hline
\end{tabular}


En las cuestiones sobre la valoración del recurso, se pregunta a los participantes por la calidad y por la fiabilidad de la información contenida en el texto como aparece en la Figura 1.

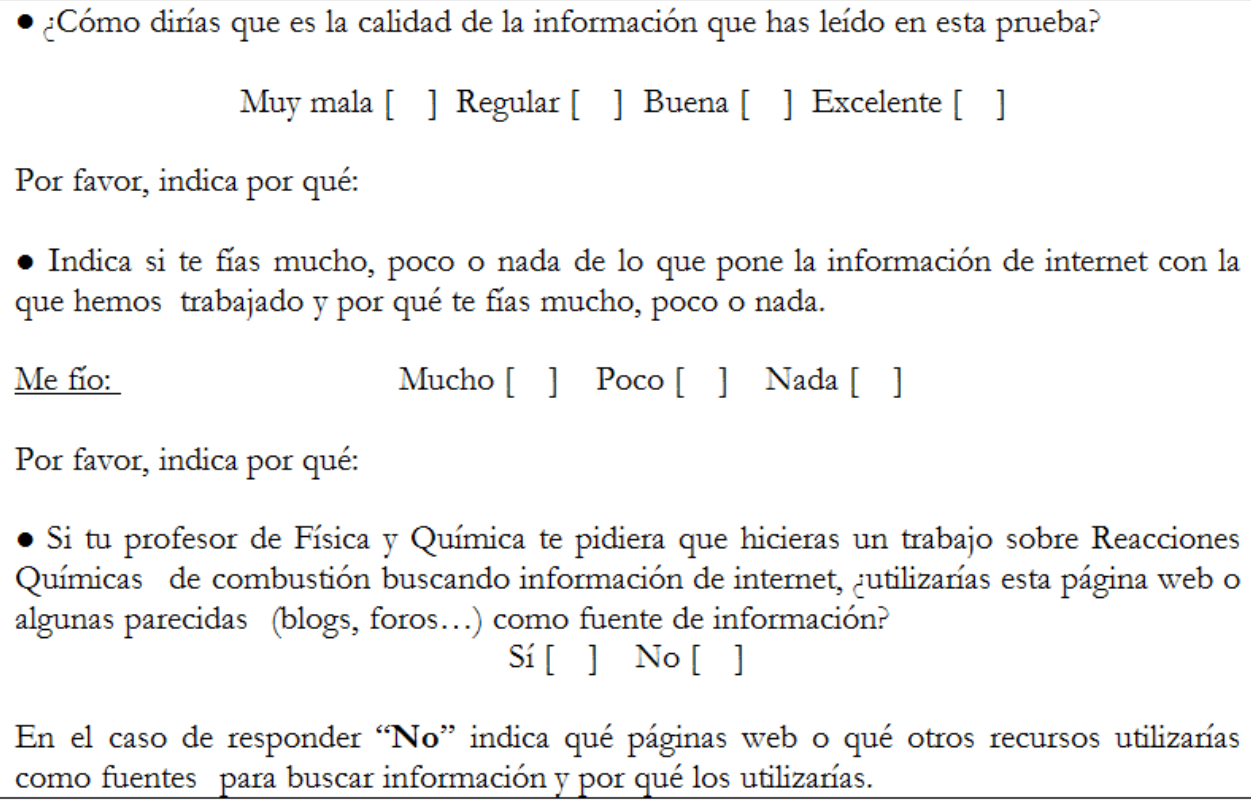

En el caso de responder "No" indica qué páginas web o qué otros recursos utilizarías como fuentes para buscar información y por qué los utilizarías.

Figura 1. Formato de cuestiones sobre calidad y fiabilidad del texto.

Una vez recogida la información, procedimos a identificar cada idea que habían plasmado los estudiantes en sus respuestas al cuestionario; cada una de ellas constituyó una unidad de información y la incluimos en sus respectivas subcompetencias de referencia. Luego procedimos a un análisis descriptivo en términos de frecuencias.

Por último, hemos de señalar que, aunque no era un objetivo del trabajo, explicamos y justificamos a los participantes todos los errores del texto y comentamos la publicidad presente después de la recogida de información, dado el interés que había despertado la actividad.

\section{Descripción de resultados}

Para describir los resultados, hemos utilizado como referentes cada una de las subcompetencias informativas digitales objeto de estudio.

\section{En relación a la subcompetencia identificación de ideas en el texto}

La identificación de ideas en el texto digital (cuestiones 1 y 2) ha obtenido resultados positivos ya que los participantes parecen habituados a localizar información literal en diferentes recursos (como apuntes, libros de texto...). Los resultados se recogen en la Tabla 2.

Tabla 2. Resultados de las Cuestiones 1 y 2.

\begin{tabular}{|l|c|c|}
\hline Identificación de ideas & $\begin{array}{c}\text { Frecuencia Cuestión 1 } \\
(\mathrm{N}=86)\end{array}$ & $\begin{array}{c}\text { Frecuencia Cuestión 2 } \\
(\mathrm{N}=86)\end{array}$ \\
\hline Identificación adecuada & 31 & 55 \\
\hline Identificación parcialmente adecuada & 36 & - \\
\hline Identificación no adecuada & 19 & 31 \\
\hline
\end{tabular}

En relación con la Cuestión 1, en las respuestas adecuadas se identificaron las ideas fundamentales: la reacción entre una sustancia combustible y oxígeno para producir productos; que éstos son sustancias diferentes a las iniciales; y la producción de energía térmica en el proceso. Algunos ejemplos, que recogen casi la literalidad del texto, son: 
- «Son procesos muy comunes en los que una sustancia combustible reacciona con oxígeno para producir otras sustancias diferentes, productos, y, generalmente, energía térmica que podemos consumir o utilizar.» (A10, A13, A31, A32, A44, etc.)

- «Es un proceso por el cual obtenemos energía térmica, al hacer reaccionar una sustancia combustible con oxígeno para producir otra diferente (un producto)» (A71)

- «Una reacción química de combustión es un proceso mediante el cual una sustancia combustible reacciona con oxígeno (llamados éstos reactivos), como resultado de la reacción se producen otras sustancias (que llamamos productos) y también se desprende energía calorífica que puede ser usada.» (A35)

En las respuestas parcialmente adecuadas se omite alguna de las ideas, normalmente las referidas al desprendimiento de energía y los cambios en la temperatura (33/36).

- «Es un proceso muy común donde una sustancia combustible reacciona al añadirle oxígeno $\left(\mathrm{O}_{2}\right)$ para producir otras sustancias.» (A1, A75, etc.)

Por su parte, entre las no adecuadas (19/86), las más frecuentes fueron las que no indicaban la generación de nuevas sustancias como parte del proceso químico $(7 / 19)$ o identificaban la energía desprendida como una sustancia producida (10/19). Ejemplos de ello son:

- «Son procesos muy comunes en los que una sustancia combustible reacciona con oxígeno para producir otras sustancias diferentes, la más común, energía térmica» (A8)

- «Son procesos en los que una sustancia combustible al juntarse con oxígeno reacciona para dar lugar a otras sustancias que normalmente suele ser energía térmica» (A72)

Respecto a la Cuestión 2, la mayoría de los participantes (55/86) identificó la información requerida («sustancia combustible y oxígeno»).

En cuanto a las respuestas no adecuadas (31/86), hubo una gran variedad. Solían identificar otras sustancias que se mencionaban en el texto pero que no coincidían con la deseable. Las más repetidas fueron «reactivos y productos» (12/31) y «azufre y nitrógeno» (6/31).

Hemos de indicar que, aunque los estudiantes no tuvieran claro lo que es una reacción química de combustión, la respuesta estaba explícita en el texto.

\section{En relación a la subcompetencia realización de inferencias lejanas al texto}

En la Cuestión 3, los estudiantes debían citar dos situaciones en las que se dieran reacciones químicas de combustión (aparecían dos en el texto a modo de ejemplo).

Globalmente, como se muestra en la Tabla 3, un amplio grupo de participantes fue capaz de responder al menos una situación donde reconocían la presencia de una reacción química de combustión de forma adecuada. Sin embargo, también se obtuvo un número elevado de respuestas en blanco. Esto refleja la dificultad para trasladar los conceptos científicos escolares a un contexto real y cotidiano.

Tabla 3. Resultados de la Cuestión 3.

\begin{tabular}{|l|c|}
\hline Número de participantes que: & Frecuencia $(\mathrm{N}=86)$ \\
\hline Hacen inferencia a 2 ejemplos adecuados & 24 \\
\hline Hacen inferencia a 1 ejemplo adecuado & 33 \\
\hline No infieren a ningún ejemplo adecuado & 5 \\
\hline Responden en blanco & 24 \\
\hline
\end{tabular}

De este modo, se recogieron 99 inferencias de 192 posibles. En la Tabla 4 se recogen los resultados en cuanto a la adecuación de las inferencias realizadas. 
Tabla 4. Resultados de la Cuestión 3.

\begin{tabular}{|l|c|}
\hline Realización de inferencias lejanas & Frecuencia (N=99) \\
\hline Inferencias lejanas adecuadas & 81 \\
\hline Inferencias lejanas ambiguas & 2 \\
\hline Inferencias lejanas no adecuadas & 16 \\
\hline
\end{tabular}

Las inferencias adecuadas fueron 81/99, siendo las más citadas: «chimenea/hoguera/barbacoa de carbón/estufa» (25/81), «mechero/vela/soplete» (23/81), «hornillos de cocina/de camping» (9/81); «locomotora de carbón» (5/81), etc.

Entre los ejemplos no adecuados se mencionan cambios físicos («aerosol en espray», «mezcla de productos químicos», «agua hirviendo»...) y otras situaciones imprecisas («aire acondicionado», «cuando el aceite toca la vitro caliente», etc.).

\section{En relación a la subcompetencia posicionamiento argumentado}

En la Cuestión 4, los estudiantes debían usar sus creencias sobre la causa de que se realicen actividades que implican combustiones si suponen un problema ambiental. Los resultados se muestran en la Tabla 5.

Tabla 5. Resultados de la Cuestión 4.

\begin{tabular}{|l|c|}
\hline Posicionamiento argumentado & Frecuencia $(\mathrm{N}=86)$ \\
\hline Respuestas adecuadas & 37 \\
\hline Respuestas parcialmente adecuadas & 42 \\
\hline Respuestas no adecuadas & 5 \\
\hline No contestan & 2 \\
\hline
\end{tabular}

Algo menos de la mitad (37/86) se apoyaron en el texto y emitieron una opinión, una creencia o un juicio al respecto que consideramos adecuados. Se utilizaron 98 argumentos diferentes, ya que algunos estudiantes utilizaron más de uno. Entre los más utilizados (63/98) encontramos la necesidad de realizar actividades cotidianas o las comodidades que precisan estas reacciones (relacionadas con el trasporte, uso de vehículos, la cocina, la higiene...).

- «Porque muchas de ellas son necesarias para la vida cotidiana, como el calentador lo necesitamos al ducharnos, y la gasolina para desplazarnos» (A89)

- «Porque necesitamos desplazarnos con vehículos y muchas veces no nos tomamos en serio el daño que pueden provocar estas sustancias al medio ambiente» (A31)

- «Porque las personas preferimos vivir cómodamente en el sentido de cuando hace frío pongo el calefactor, coger el coche para desplazarnos a sitios donde podríamos ir andando...» (A66)

También se citan causas de tipo económico (20/98), como que introducir fuentes de energía renovables y limpias sería costoso, o la influencia de la industria del petróleo.

- «Porque hay que usar fuentes de energía renovables, aunque sean más costosas» (A62)

- «Porque hay que cambiar los coches de combustibles fósiles por eléctricos» (A67)

- «Porque es muy difícil reducir el uso de los combustibles fósiles» (A13)

En menor medida, se citan causas de tipo social (9/98) como la desinformación o una falta de concienciación ambiental.

- «Porque la sociedad está poco informada sobre lo perjudiciales que resultan a la atmósfera» (A11)

- «Porque la ignorancia humana prefiere su comodidad y el dinero antes que no contaminar su planeta» (A57) 
- «Porque, aunque sean muy perjudiciales, tienen efectos rápidos y eficaces y como en el momento que lo estamos utilizando no tiene efectos inmediatos no somos suficientemente conscientes de la situación y los peligros que conlleva» (A17)

Sin embargo, hay muchas respuestas que se han considerado parcialmente adecuadas $(42 / 86)$ ya que, aunque se basan en la información del texto para posicionarse, la brevedad de su redacción o la baja calidad de sus argumentos, no permiten explicitar con claridad sus opiniones o juicios («Porque las necesitamos en la vida diaria», «Porque hay que ahorrar energía», etc.).

\section{En relación a la subcompetencia localización de errores en el texto}

En la Cuestión 5 se pedía que localizaran y justificaran tres errores presentes en el texto. Casi todos (84/86) la respondieron, pero se recogieron 138 errores de los 258 posibles, ya que la mayoría (78/84) localizó menos de tres errores. Los resultados se sintetizan en la Tabla 6.

Tabla 6. Resultados de la Cuestión 5.

\begin{tabular}{|c|c|}
\hline Localización de errores & Justificación de errores localizados \\
\hline Errores totales localizados: 138 & $\begin{array}{l}\text { Errores totales justificados: } 129 \\
\text { Errores no justificados: } 9\end{array}$ \\
\hline $\begin{aligned} \text { Localización adecuada: } 119 \\
\text { - } \quad \text { Localiza error 1: } 24 \\
\text { - } \quad \text { Localiza error 2: } 75 \\
\text { - Localiza error 3: } 20 \\
\end{aligned}$ & $\begin{array}{l}\text { Justificación de forma adecuada: } 78 \\
\text { - Justifica de forma adecuada error 1: } 22 \\
\text { - Justifica de forma adecuada error 2: } 48 \\
\text { - Justifica de forma adecuada error 3: } 8\end{array}$ \\
\hline Localización no adecuada: 19 & Justificación no adecuada: 51 \\
\hline
\end{tabular}

Como se observa, a pesar de que se ha localizado un número bajo de errores, la identificación ha sido adecuada en un porcentaje alto de ocasiones (119/138). El error 2 (nomenclatura errónea para el $\mathrm{CO}_{2}$ ) ha sido el más localizado (por 75/86 participantes), mientras que los otros (emisión de $\mathrm{CO}_{2}$ por habitante y confusión reactivos-productos) lo han sido sólo por el $25 \%$ del alumnado aproximadamente.

Los participantes encontraron más dificultad en la justificación de los errores que localizaban, ya que de los 119 errores localizados correctamente sólo 78 fueron explicados adecuadamente.

El error 1 (reactivos y productos confundidos en una ecuación química) fue justificado de forma correcta por 22/24 de los que lo habían localizado. Algunos ejemplos son:

- «Porque los reactivos son las sustancias que reaccionan y los productos las sustancias resultantes/ resultado final» (A13, A23, A77, etc.)

- «Porque los productos y reactivos están al revés/está cambiado el orden» (A17, A19, A22, A39, A44, A71, etc.)

En el error 2, 48/75 justificaciones las consideramos adecuadas:

- «En la combustión de hidrocarburos se produce dióxido de carbono, no trióxido» (A24, A27, A79, etc.)

- «Es/Se refiere a dióxido de carbono» (A3, A5, A9, A13, etc.)

A éstas se podría añadir otras (13/75), que detectan la nomenclatura del compuesto, pero no se centran en el contenido de la información, por ello no las consideramos adecuadas:

- «Debería escribirse $\mathrm{CO}_{3} /$ Para que la fórmula coincidiera tendría que ser $\mathrm{CO}_{3} »$ (A20, A38, A73, etc.) 
Finalmente, en el error 3, sólo 8/20 justificaciones las consideramos adecuadas:

- «La cantidad de emisiones descrita en el texto es demasiado pequeña para ser un problema ambiental/ demasiado pequeño para preocuparse por ello» (A35, A46)

- «Se emite mucho más/más de 0,0000058 kg por persona» (A52, A68)

- «Se emitieron/Serían $5800 \mathrm{~kg}$, no 0,0000058 kg/ Fueron más de 13 millones de toneladas en toda España en 2011» (A39, A44, A40, A51, aportan otro dato que han buscado y encontrado en Internet)

Hay otras respuestas que se centran en que la unidad no es correcta: «Porque el $\mathrm{CO}_{2}$ no se mide en kilogramos», «Los gases no se miden en kilos», etc.; las consideramos no adecuadas.

En resumen, sólo 3/86 estudiantes fueron capaces de localizar y justificar adecuadamente los tres errores (A39, A44 y A46). Esto puede ser debido a que los conocimientos influyen en la justificación (Colwell et al. 2013) y, probablemente por ello, les resultó difícil razonar con argumentos de carácter científico los errores que habían detectado.

\section{En relación a la subcompetencia interpretación de la intencionalidad de información}

En la Cuestión 6 se pedía interpretar el contenido del último párrafo del texto, en el que se realiza publicidad de un producto (ficticio). Los resultados se muestran en la Tabla 7.

Tabla 7. Resultados de la Cuestión 6.

\begin{tabular}{|l|c|}
\hline Interpretación de información & Frecuencia $(\mathrm{N}=86)$ \\
\hline Interpretación adecuada & 44 \\
\hline Interpretación parcialmente adecuada & 14 \\
\hline Interpretación no adecuada & 26 \\
\hline No contestan & 2 \\
\hline
\end{tabular}

Los estudiantes hicieron una interpretación adecuada en muchas respuestas (44/84), identificando que se hace publicidad de un producto, y lo justificaron apoyándose en la información del texto.

- «Concienciar a la gente de lo perjudiciales que son los gases y aprovecha el último párrafo para publicitar su producto como la mejor solución. Porque saca el tema de la contaminación y con su producto al parecer se evita» (A1).

- «Lo que quiere es dar publicidad para que compremos el filtro. La última frase es como la de un anuncio, parece más publicidad que otra cosa» (A36)

- «La autora pretende venderte el producto que anuncia/que ha fabricado la empresa en la que trabaja. Te dice las características y su precio» (A63, A85).

Consideramos parcialmente adecuadas las respuestas que interpretaron bien la información, pero no la justificaron suficientemente, al no incidir en el carácter publicitario y persuasivo del texto.

- «Explicar las principales causas de las emisiones por la combustión y así mentalizarnos de lo perjudicial que es. Porque nos estamos cargando el planeta.» (A8)

- «Que nos concienciemos y utilicemos métodos para reducir la contaminación del medio, como usando filtros para los tubos de escape. Porque si la gente lo lee va a conseguir que lo cumplamos.» (A30)

- «Pretende concienciarnos de que el efecto invernadero está afectando profundamente y que por muy poco podemos hacer grandes cambios y cuidar el medio ambiente, porque en un futuro seremos nosotros los perjudicados.» (A81) 
En el caso de las respuestas no adecuadas, se realizaron interpretaciones consideradas incorrectas que se centraban en otros párrafos del texto y no en el que se les pedía.

\section{En relación a la calidad y la fiabilidad de la información}

La Figura 2 muestra los resultados recogidos en las cuestiones que piden a los participantes que valoren la calidad de la información y su fiabilidad, justificando sus respuestas.

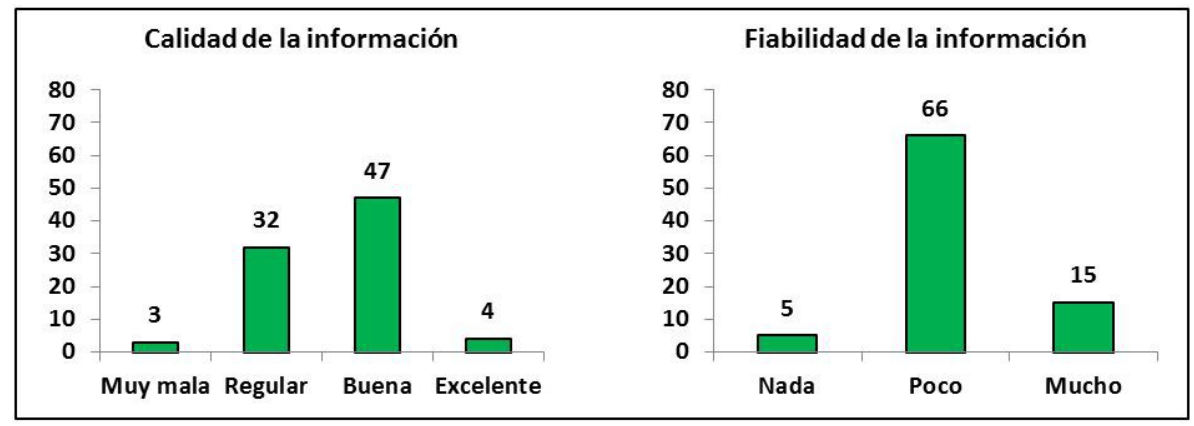

Figura 2. Respuestas sobre la calidad y la fiabilidad de la información del texto.

En cuanto a la calidad, se observa que las respuestas más recogidas son «Buena» (47/86) y «Regular» (32/86). Destaca que la más frecuente sea valorarla como «Buena», a pesar de saber de la existencia de 3 errores y de que muchos de ellos interpretaran correctamente la finalidad de la publicidad.

Entre los que la calificaron de buena calidad, justificaron que se debía a su inteligibilidad (15/47): «estaba bien expresada», «era clara», «se entendía todo»... Otros se basaron en la cantidad de información (6/47) o en la presencia de ejemplos (6/47). Y los hay (8/47) que añadieron calificativos superficiales, por ejemplo: «precisa», «detallada» o «completa»; resulta preocupante que califiquen de esa forma un texto que, según les decíamos, contiene errores.

Entre los que valoraron regular la información, se basaron en la presencia de errores (22/32) y, en menor medida, en la existencia de publicidad (9/32).

Respecto a la fiabilidad, la mayoría respondió fiarse «poco» (66/86) de la información. Para justificarlo aludieron a: la presencia de errores (29/66), la presencia de publicidad (14/66), el recurso utilizado (blogs e Internet, 9/66), la autoría de la información (5/66), etc.

Aun así, un grupo respondió que se fiaban «mucho» (15/86); de ellos, 5/15 no indicaron las causas de esa apreciación. Entre los que se fiaban, hablaban de: «Debido a que había errores (hechos a posta) para completar los ejercicios, las fórmulas, etc., están hechas bien» (A1); «Porque me parece muy aceptable para nuestra edad y da información que nosotros ya sabemos» (A12); «Porque pone mucha información en pocos párrafos, está bien resumida y adaptada a nuestro nivel» (A37), etc.

Por último, se preguntó a los participantes si utilizarían el texto si tuvieran que buscar información sobre reacciones químicas. La mayor parte respondió que sí (47/86) lo que contrasta con la fiabilidad que le habían otorgado.

Entre los que no lo utilizarían (39/86), citan recursos alternativos: Wikipedia y Google (como fuente de páginas aleatorias). También mencionaron «enciclopedias» o «el libro de física y química» mostrando que sigue existiendo una alta confianza en los recursos tradicionales.

\section{Relación entre las variables estudiadas}

Por último, hemos estudiado la relación entre los valores obtenidos en las subcompetencias, mediante el coeficiente @ de Spearman. En la Tabla 8, se recogen los valores significativos. 
Tabla 8. Relación entre subcompetencias.

\begin{tabular}{|c|c|c|c|c|c|c|}
\hline & $\begin{array}{c}\text { Identificació } \\
\text { n de Ideas } \\
\text { (IdI) }\end{array}$ & $\begin{array}{c}\text { Inferencias } \\
\text { Lejanas } \\
\text { (InLej) }\end{array}$ & $\begin{array}{c}\text { Posicionamiento } \\
\text { Argumentado } \\
\text { (PA) }\end{array}$ & $\begin{array}{c}\text { Localización } \\
\text { de errores } \\
\text { (LocE) }\end{array}$ & $\begin{array}{c}\text { Justificación } \\
\text { de errores } \\
\text { (JustE) }\end{array}$ & $\begin{array}{c}\text { Interpretación de } \\
\text { la } \\
\text { Intencionalidad } \\
\text { (InI) }\end{array}$ \\
\hline IdI & 1 & ns & $\begin{array}{c}\varrho=0,28 \\
\text { (sig.=.01) }\end{array}$ & $\begin{array}{c}\varrho=0,26 \\
\text { (sig. }=.02)\end{array}$ & ns & ns \\
\hline InLej & & 1 & $\mathrm{~ns}$ & $\mathrm{~ns}$ & ns & $\mathrm{ns}$ \\
\hline PA & & & 1 & ns & $\mathrm{ns}$ & ns \\
\hline LocE & & & & 1 & $\begin{array}{c}\varrho=0,76 \\
(\text { sig. }=.00)\end{array}$ & ns \\
\hline JustE & & & & & 1 & $\mathrm{~ns}$ \\
\hline Ini & & & & & & 1 \\
\hline
\end{tabular}

Se producen relaciones estadísticamente significativas entre «Identificación de ideas» y «Posicionamiento argumentado» y «Localización de errores». También la hay entre «Localización de errores» y «Justificación de errores»; esta última relación tiene una cierta lógica porque la justificación está supeditada a la localización.

Por último, no encontramos relaciones estadísticamente significativas entre calidad y fiabilidad, y ninguna de las subcompetencias estudiadas.

\section{Consideraciones finales}

En primer lugar, señalamos que, en este trabajo, pretendíamos estudiar cómo unos estudiantes utilizaban su competencia digital para valorar una información de Internet sobre un tema científico. En este caso se decidió utilizar un texto que presentase problemas de fiabilidad.

La información textual utilizada ha requerido que los participantes pongan en juego unas subcompetencias digitales del área de información. De este modo, podemos decir que:

- Los resultados más positivos se producen en la «identificación de ideas en el texto»y en el «posicionamiento argumentado». La menor exigencia cognitiva de estas cuestiones y un mayor hábito en su realización (en especial la primera) pueden justificar estos resultados.

- Respecto a la «interpretación de la intencionalidad de la información» se obtienen resultados irregulares ya que sólo la mitad de los participantes detecta la presencia de la publicidad y se apoya en el contenido para justificar su respuesta.

- En la «realización de inferencias lejanas» se obtienen resultados inadecuados ya que más de la cuarta parte de los participantes no responde esta pregunta (a pesar de que en el texto se daban algunos ejemplos aclaratorios). Esto indica que los conocimientos científicos escolares siguen estando alejados de su realidad cotidiana.

- También se dan resultados no deseables en la «localización y justificación de errores». Además de localizar menos de los que se podía esperar, sólo 3/86 estudiantes responden de forma totalmente adecuada. Es posible que en muchos casos solamente los localicen por lo llamativos que resultan. Además, se observan patrones diferentes en función del tipo de error (textual, numérico, gráfico...), pero no podemos indagar al respecto con los datos de los que disponemos. 
- Hemos observado una relación significativa entre los resultados de «identificación de ideas» y «posicionamiento argumentado» y «localización de errores», estas tres subcompetencias tienen una característica común que la respuesta deseable se apoya, en gran parte, en el texto. Las demás parece que están más condicionadas por el conocimiento que el estudiante tenga de la temática que por el uso que hace de las ideas presentes o contenidas en el documento.

- Encontramos incoherencias al valorar la calidad y la fiabilidad del texto, lo que nos genera especial preocupación. A pesar de que la mayoría de los estudiantes dice fiarse poco del texto, un número importante califica como buena su calidad o afirma que lo utilizarían en una tarea escolar.

Como hemos señalado, las subcompetencias donde han tenido más dificultades han sido la localización y justificación de errores y las inferencias lejanas, probablemente porque son mayores sus exigencias cognitivas, es decir, van más allá del propio texto y demandan la utilización del conocimiento que el estudiante tiene sobre la temática. Esta idea viene recogida en el marco de la argumentación científica de Toulmin (Jiménez-Aleixandre, Gallástegui, Eirexas y Puig 2009, Jiménez-Aleixandre 2010) y apoya la idea de que la adquisición de la competencia digital requiere un tratamiento integrado entre las destrezas del área de evaluación y análisis de información con otras competencias que implican el conocimiento de la Ciencia para que realmente incida en ésta.

Las dificultades e incoherencias mostradas ponen de manifiesto que nuestros participantes no disponen de procedimientos que les permitan realizar una evaluación crítica de un texto de Internet. Observamos que el desarrollo de sus competencias digitales, a pesar de su contacto diario con las tecnologías fuera del aula, no es suficiente; necesitan una formación intencionada, tal como reconocen otros estudios (Calvani et al., 2012, Hatlevik y Christophersen 2013).

Durante la realización de la experiencia los participantes, en su mayoría, se mostraron dubitativos e inseguros en varias de las cuestiones, especialmente al localizar y justificar errores y al hacer inferencia a situaciones donde identificaran reacciones químicas. Del mismo modo, a la hora de valorar la fiabilidad y calidad mostraron dificultades y, con frecuencia, dieron respuestas escuetas, superficiales y, en algunos casos contradictorias. Esto nos permite interpretar que no están habituados a este tipo de tareas y, de acuerdo a aportaciones previas (Julien y Barker 2009, Walraven et al. 2009), no suelen dudar de los contenidos espontáneamente. Ha sido necesario introducir errores y publicidad para motivarlos a ello. En este sentido, nuestros resultados, especialmente las incoherencias y dificultades que han mostrado a la hora de valorar calidad y fiabilidad, apuntan a que las ideas que pueden tener los estudiantes sobre «información de calidad» se centren en aspectos tales como la claridad, la expresión, la cantidad de información o la presencia de ejemplos, y no tanto a la presencia o ausencia de errores, publicidad, etc.

De este modo, podemos concluir que los estudiantes pueden ser sujetos vulnerables a la transmisión de información basura, publicitaria, bulos o de cuestionable calidad que abunda en la red. Por ello, es necesario un tratamiento escolar complementario entre contenidos científicos y diferentes competencias (en este caso digitales) para facilitar una formación básica e integral para todo ciudadano que le permita hacer frente a los problemas informacionales que existen hoy en la red, y que todo parece indicar que en un futuro próximo continuarán existiendo.

Disponemos de algunos ejemplos de propuestas actuales al respecto, como pueden ser los trabajos de Franco, España y Blanco (2014), Franco (2015) o Blanco, España y Franco (2017), 
donde se trabajan contenidos científicos inmersos en temáticas relevantes a la vez que capacidades de tipo digital, entre otras, pero aún resultan insuficientes en número. En este sentido, la propia experiencia planteada, con su justificación y ejemplificación de subcompetencias relevantes del área de «Evaluación de la información», podría ser un punto de partida útil para diseñar e incorporar otras actividades que trabajen la competencia digital en el ámbito del aprendizaje de las ciencias.

Entre las limitaciones de este trabajo encontramos que la experiencia se ha realizado con un solo recurso y con unos errores determinados. Para describir la competencia digital del alumnado al evaluar información será necesario la realización de estudios que permitan una descripción más amplia, que incluya más recursos y otros formatos como animaciones, simulaciones, vídeos, publicidad y noticias o informaciones transmitidas a través de redes sociales (Valverde-Crespo, Pro-Bueno y González-Sánchez 2018).

\section{Referencias}

Area M., Cepeda O., Romero L. (2018) El uso escolar de las TIC desde la visión del alumnado de Educación Primaria, Secundaria y Bachillerato. Educatio Siglo XXI, 36(2), 229-254.

Banco Mundial (2016). Emisiones de CO2 (toneladas métricas per cápita).

Blanco A., España E., Franco A.J. (2017) Estrategias didácticas para el desarrollo del pensamiento crítico en el aula de ciencias. Ápice. Revista de Educación Científica, 1(1), $107-$ 115.

BOE (2015) Orden ECD/65/2015, de 21 de enero, por la que se describen las relaciones entre las competencias, los contenidos y los criterios de evaluación de la educación primaria, la educación secundaria obligatoria y el bachillerato. Recuperado de: https://www.boe.es/buscar/doc.php?id=BOE-A-2015-738

Bravo B., Bouciguez M. J., Braunmüller M. (2019) Una propuesta didáctica diseñada para favorecer el aprendizaje de la Inducción Electromagnética básica y el desarrollo de competencias digitales. Revista Eureka sobre Enseñanza y Divulgación de las Ciencias, 16(1), 1203.

Caamaño A. (1998) El cambio químico: un tema central de la investigación en didáctica de la química. Alambique: Didáctica de las Ciencias Experimentales, 17, 61-64.

Calvani A., Fini A., Ranieri M., Picci P. (2012) Are young generations in secondary school digitally competent? A study on Italian teenagers. Computers \& Education, 58, 797-807.

Cantó-Doménech J., Pro-Bueno A., Solbes J. (2017) ¿Cómo utilizan los conocimientos en ciencias los futuros maestros de educación infantil ante una información escrita? Didáctica de las Ciencias Experimentales y Sociales, 33, 99-122.

CARM (2015) Decreto número 220/2015, de 2 de septiembre, por el que se establece el currículo de la Educación Secundaria Obligatoria en la Comunidad Autónoma de la Región de Murcia. Boletín número 203, de 3 de septiembre de 2015. Murcia: CARM.

Colás P., Conde J. Reyes S. (2017) Competencias digitales del alumnado no universitario. RELATEC. Revista Latinoamericana de Tecnología Educativa, 16(1), 7-20.

Colwell J., Hunt S. Reinking D. (2013) Obstacles to Developing Digital Literacy on the Internet in Middle School Science Instruction. Journal of Literacy Research, 45(3), 295324. 
EFE (12 de febrero 2018) Uno de cada tres bulos que circulan por Internet es sobre salud. Recuperado de: La Vanguardia

FECYT (2015) VII Encuesta de percepción social de la ciencia. Dossier informativo.

Fernández J.P. (2016) La adquisición y desarrollo de la competencia digital en alumnos de educación secundaria. Un estudio de caso. Cuadernos de Investigación Educativa, 7(2), 8398.

Ferrari A. (2013) DIGCOMP: A Framework for Developing and Understanding Digital Competence in Europe. En Y. Punie, y B.N. Brecko (Eds.), JRC scientific and policy reports. Louxembourg: Publications Office of the European Union.

Forzani E. (2018) How Well Can Students Evaluate Online Science Information? Contributions of Prior Knowledge, Gender, Socioeconomic Status, and Offline Reading Ability. Reading Research Quarterly, 53(4), 385-390.

Franco A.J., España E. Blanco A. (2014) Uso de Internet para analizar las relaciones entre drogas y salud bucodental. Una experiencia en $4^{\circ}$ de ESO. En Soriano, E.; González, A.J. y Cala, V.C. (eds.). Retos actuales de educación y salud transcultural [1]. Almería: Universidad de Almería, cap. 54.

Franco A.J. (2015) Competencias científicas en la enseñanza y el aprendizaje por investigación. Un estudio de caso sobre corrosión de metales en secundaria. Enseñanza de las Ciencias, 33(2), 231-252.

Gómez M.A, Cañas A.J., Gutiérrez M.S. Martín M.J. (2014) Ordenadores en el aula: ¿estamos preparados los profesores? Enseñanza de las Ciencias, 32(2), 239-250.

Grimalt C., Pintó R. Ametller J. (2013) La utilización del aula digital en las clases de ciencias de secundaria. Análisis del estado actual. Proyecto ADIGIC. Alambique: Didáctica de las Ciencias Experimentales, 76, 91-98.

Hatlevik O.E., Christophersen K.A. (2013) Digital competence at the beginning of upper secondary school: Indetifying factors explaining digital inclusion. Computers \& Education, 63, 240-247.

Jiménez-Aleixandre M.P., Gallástegui J.R., Eirexas F., Puig B. (2009) Actividades para trabajar el uso de pruebas y la argumentación en ciencias. Santiago de Compostela: Danú.

Jiménez-Aleixandre, M.P. (2010) 10 ideas clave. Competencias en argumentación y uso de pruebas. Barcelona: Graó.

Jiménez R. (22 de septiembre de 2017) Facebook, contra las noticias falsas en España. Recuperado de: El País

Julien H., Barker S. (2009) How high-school students find and evaluate scientific information: A basis for information literacy skills development. Library \& Information Science Research, 31, 12-17.

Keck D., Kammerer Y., Starauschek E. (2015) Reading science text online: Does source information influence the identification of contradictions within texts? Computers \& Education, 82, 442-449.

Lacolla L., Meneses J.A., Valeiras, N. (2013) Las representaciones sociales y las reacciones químicas: Desde las explosiones hasta Fukushima. Educación Química, 24(3), 309-315.

Li Y., Ranieri M. (2010) Are 'digital natives' really digitally competent?-A study on Chinese teenagers. British Journal of Educational Technology, 41(6), 1029-1042. 
Mason L., Junyent A., Tornatora M.C. (2014) Epistemic evaluation and comprehension of web-source information on controversial science-related topics: Effects of a short-term instructional intervention. Computers \& Education, 76,143-157.

Monereo C. (2005) Internet y competencias básicas: Aprender a colaborar, a comunicarse, a aprender. Barcelona: Graó.

OECD (2016) Air and GHG emissions.

Olazabal P., Echeverría J. (2013) La televisión e Internet como herramientas de aprendizaje y desarrollo de la capacidad crítica en el alumnado. Enseñanza de las Ciencias, $\mathrm{N}^{\circ}$ Monográfico: IX Congreso Internacional sobre Investigación en Didáctica de las Ciencias, 990-996.

Otero J. C., Campanario J. (1990) Comprehension, evaluation and regulation in learning from science texts. Journal of Research in Science Teaching, 27(5), 447-460.

Pedrinaci E. (2012a) El ejercicio de una ciudadanía responsable exige disponer de cierta competencia científica. En Pedrinaci, E. (coord.) 11 ideas clave. El desarrollo de la competencia científica, (pp. 15-35). Barcelona: Graó.

Pedrinaci E. (2012b) Aprender ciencias es, en buena medida, aprender a leer, escribir y hablar ciencia. En Pedrinaci E. (coord.) 11 ideas clave. El desarrollo de la competencia cientifica (pp. 147-169). Barcelona: Graó.

Pereira S., Fillol J., Moura P. (2019) El aprendizaje de los jóvenes con medios digitales fuera de la escuela: De lo informal a lo formal. Comunicar, 58, 41-50.

Pro-Bueno A. (2014) ¿Cómo son las actividades de enseñanza planteadas para enseñar la energía? En Pro-Bueno A., La energía: uso, consumo y ahorro energético en la vida cotidiana (pp. 77-123). Barcelona: Graó.

Revuelta G., Corchero C. (2017) Perfiles generacionales en el consumo de información científica. En FECYT (Ed.) Percepción Social de la Ciencia y la Tecnología 2016, (pp. 179206). Madrid: MIC.

Solsona N., Izquierdo M. (1999) El aprendizaje del concepto de cambio químico en el alumnado de secundaria. Investigación en la Escuela, 38, 65-75.

Somyurek S., Coskun B. (2013) Digital competence: Is it an innate talent of the new generation or an ability that must be developed? British Journal of Educational Technology, 44(5), 163-166.

Sullivan S., Puntambekar S. (2019) Learning with multiple online texts as part of scientific inquiry in the classroom. Computers \& Education, 128, 36-51.

Valverde-Crespo D., González-Sánchez J. (2016) Búsqueda y selección de información en recursos digitales: Percepciones de alumnos de Física y Química de Educación Secundaria Obligatoria y Bachillerato sobre Wikipedia. Revista Eureka sobre Enseñanza y Divulgación de las Ciencias, 13(1), 67-83.

Valverde-Crespo D., González-Sánchez J., Pro-Bueno A. (2017) ¿Qué sub-competencias digitales muestran unos alumnos de $4^{\circ}$ de Educación Secundaria Obligatoria ante una animación sobre una reacción química a nivel microscópico?. Ápice. Revista de Educación Cientifica, 1(1), 40-57.

Valverde-Crespo D., Pro-Bueno A., González-Sánchez J. (2018) La competencia informacional-digital en la enseñanza y aprendizaje de las ciencias en la educación 
secundaria obligatoria actual: una revisión teórica. Revista Eureka sobre Enseñanza y Divulgación de las Ciencias, 15(2), 2105.

Walraven A., Brand-Gruwel S., Boshuizen H. (2009) How students evaluate information and sources when searching the World Wide Web for information. Computers \& Education, 52, 234-236. 


\section{Anexo I}

Texto empleado para la investigación

\section{REACCIONES QUÍMICAS DE COMBUSTIÓN.}

Las reacciones químicas de combustión son procesos muy comunes en los que una sustancia combustible reacciona con oxígeno (reactivos) y producen otras sustancias diferentes (productos) y, generalmente, energía térmica que podemos consumir o utilizar.

Ejemplos muy clásicos son la combustión de los hidrocarburos como la gasolina en los motores, o el que ponemos a continuación de la combustión del butano $\left(\mathrm{C}_{4} \mathrm{H}_{10}\right)$ en un calentador de agua.

$$
\begin{aligned}
& \mathrm{C}_{4} \mathrm{H}_{10}+\frac{13}{2} \mathrm{O}_{2} \rightarrow 4 \mathrm{CO}_{2}+5 \mathrm{H}_{2} \mathrm{O} \\
& \text { PRODUCTOS REACTIVOS }
\end{aligned}
$$

En la reacción de combustión de hidrocarburos se produce trióxido de carbono $\left(\mathrm{CO}_{2}\right)$ como uno de los productos, por lo que se consideran reacciones perjudiciales para el medio ambiente ya que esta sustancia es un gas de efecto invernadero. Además, cuando los combustibles que participan en reacciones químicas de combustión contienen azufre o nitrógeno, la reacción también produce óxidos de azufre y óxidos de nitrógeno que son gases que en la atmósfera provocan la formación de lluvia ácida.

Por ello debemos ser cuidadosos con nuestras actividades de consumo, transporte... porque junto con ellas ocurre una reacción química de combustión perjudicial tanto para el medio ambiente como para nosotros mismos, que en el año 2011, por ejemplo, dio lugar a emisiones en torno a los 0,0000058 kilogramos de $\mathrm{CO}_{2}$ por persona en España.

Una de las principales causas de estas emisiones ocurre por la combustión de la gasolina en los motores. Sin embargo, recientes estudios demuestran que el $\mathrm{CO}_{2}$ y otros gases procedentes de las reacciones de combustión provocadas por la actividad humana pueden ser eliminados si se coloca en el tubo de escape de tu vehículo el filtro Anti-CombustionSuper1000 (fabricado por Industrias ChemicalBogus). Así que no pierdas el tiempo y por 19,95 dólares, echa una mano a la naturaleza.

Giorgia Cannondale,

Asesora cientifico-comercial de Industrias ChemicalBogus. 